\title{
Exclusive meson production at HERMES
}

\author{
M. Tytgat ${ }^{\mathrm{a}}$ \\ On behalf of the HERMES Collaboration \\ University of Gent, Department of Subatomic and Radiation Physics, Proeftuinstraat 86, 9000 Gent, Belgium
}

Received: 8 October 2006

Published online: 21 February 2007 - C Società Italiana di Fisica / Springer-Verlag 2007

\begin{abstract}
Hard exclusive production of mesons in deep-inelastic scattering allows one to probe the so-far unknown Generalized Parton Distributions (GPDs) of the nucleon. The HERMES experiment has measured several different observables in exclusive meson production by scattering the $27.6 \mathrm{GeV}$ HERA lepton beam off an internal fixed gaseous target. Recent results on exclusive $\pi^{+}, \rho^{0}$ and pion pair production will be presented.
\end{abstract}

PACS. 13.60.-r Photon and charged-lepton interactions with hadrons - 13.60.Le Meson production 13.88.+e Polarization in interactions and scattering - 14.20.Dh Protons and neutrons

\section{Introduction}

Exclusive reactions in electron-nucleon scattering have recently gained interest within the framework of the Generalized Parton Distributions (GPDs) [1]. In the QCD factorization theorem for hard exclusive electroproduction of mesons with longitudinal photons [2], GPDs appear in the parametrization of the target nucleon. They extend our description of the nucleon spin structure beyond the form factors and standard parton distributions which we know from (semi)-inclusive deep-inelastic scattering. GPDs are sensitive to correlations between partons with different momenta and can probe the nucleon transverse and longitudinal momentum structure in a coherent manner. The second moment of these GPDs is in fact related to the total angular-momentum contribution of partons to the nucleon spin. In leading twist the nucleon can be parametrized using two unpolarized GPDs, $H$ and $E$, and two polarized ones, $\tilde{H}$ and $\tilde{E}$, where the quantum numbers of the produced final state determine the sensitivity to different GPDs.

The data presented here were collected from 1996 to 2004 with the HERMES spectrometer [3], using hydrogen and deuterium gas targets internal to the $27.6 \mathrm{GeV}$ HERA lepton storage ring at DESY.

\section{Exclusive $\pi^{+}$production}

Exclusive $\pi^{+}$production on the nucleon is sensitive to the polarized GPDs $\tilde{E}$ and $\tilde{H}$ without the need for beam or target polarization and is studied at HERMES by requiring the missing mass $M_{X}$ for the reaction $e p \rightarrow e X \pi^{+}$

\footnotetext{
${ }^{\text {a }}$ Postdoctoral Fellow of the FWO Flanders, Belgium; e-mail
} michael. tytgat@ugent.be

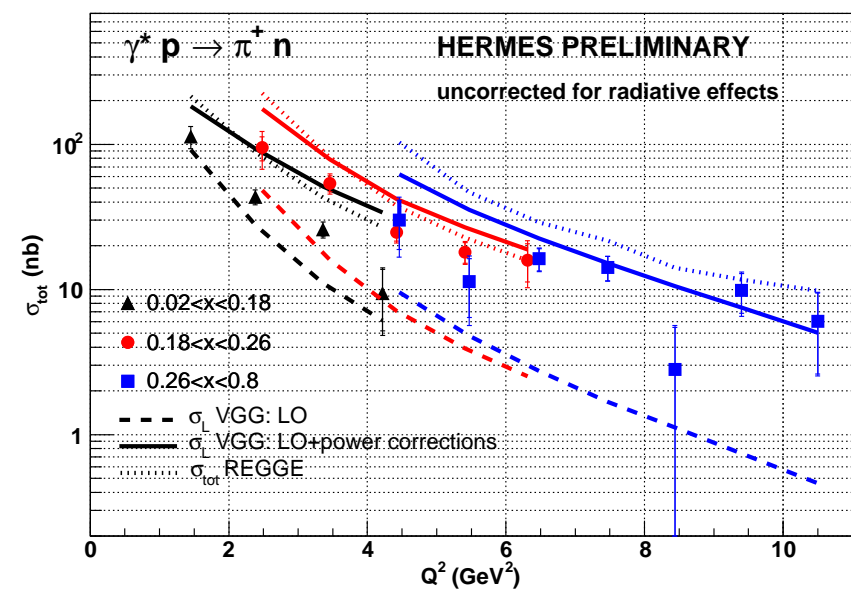

Fig. 1. The exclusive $\pi^{+}$production cross-section as a function of $Q^{2}$ for several $x_{B}$ ranges compared to GPD [4] and Regge [6] model calculations.

to be near the nucleon mass. Due to the limited resolution in $M_{X}$, the exclusive channel $e p \rightarrow e n \pi^{+}$cannot be separated easily from the neighboring channels and non-exclusive background and hence, as the process $e p \rightarrow e n \pi^{-}$is forbidden, a normalized sample of $e p \rightarrow e X \pi^{-}$events was subtracted from the data to obtain a pure exclusive $\pi^{+}$signal.

For the determination of the absolute production cross-section, the detection probability was derived using two Monte Carlo simulations for exclusive $\pi^{+}$production based on different GPD parametrizations [4,5]. The measured $Q^{2}$-dependence of the total photoproduction cross-section is shown in fig. 1 . Radiative effects have not been accounted for in this result and are estimated to 
be about $20 \%$ with little $x_{B}$ or $Q^{2}$-dependence. A separation of the cross-section into its transverse and longitudinal components is not feasible at HERMES. However, with a virtual photon polarization parameter $\epsilon$ ranging from 0.80 to 0.95 at HERMES and the predicted power suppression of the transverse part [2], the total cross-section $\sigma_{t o t}=\sigma_{T}+\epsilon \sigma_{L}$ is expected to be dominated by the longitudinal component at large $Q^{2}$. The data in fig. 1 are compared to GPD model calculations [4] for the longitudinal cross-section. While the $Q^{2}$ behavior is in agreement with the data, the leading-order calculations underestimate the cross-section, whereas including power corrections overestimates the data. The Regge model calculations [6] for the total cross-section shown in the figure are seen to overestimate the data. In the latter model the transverse part of the cross-section is suppressed with respect to the longitudinal contribution by at least a factor four. The observed $Q^{2}$ behavior of the measured cross-section is in agreement with a $1 / Q^{6}$-dependence at fixed $x_{B}$ as predicted by the factorization theorem for the longitudinal cross-section [2].

\section{Exclusive $\rho^{0}$ production}

Hard exclusive $\rho^{0}$ production provides a probe for the unpolarized GPDs $H$ and $E$. At HERMES this process can be cleanly identified by reconstructing the $\rho^{0}$ in its 2-pion decay channel and by requiring $\Delta E=\left(M_{X}^{2}-M^{2}\right) / 2 M$, which is a measure of the missing energy in the $e p \rightarrow e X \rho^{0}$ reaction, to be close to zero. Non-exclusive background in the $\rho^{0}$ event sample is corrected for using Monte Carlo simulations.

In hard exclusive $\rho^{0}$ production the transverse target single-spin asymmetry (TTSSA) is sensitive to the interference between GPD $E$ and $H$ [7]. Whereas in the crosssection GPD $E$ is kinematically suppressed with respect to GPD $H$, the asymmetry has a linear dependence on GPD $E$ and is therefore expected to provide a good handle on the latter. Furthermore, the TTSSA also appears sensitive to the total $u$-quark angular momentum [8]. The asymmetry is calculated as

$$
\begin{aligned}
A_{U T}\left(\phi, \phi_{S}\right) & =\frac{1}{\left|S_{\perp}\right|} \frac{N^{\uparrow}\left(\phi, \phi_{S}\right)-N^{\downarrow}\left(\phi, \phi_{S}\right)}{N^{\uparrow}\left(\phi, \phi_{S}\right)+N^{\downarrow}\left(\phi, \phi_{S}\right)} \\
& =A_{U T}^{\sin \left(\phi-\phi_{S}\right)} \sin \left(\phi-\phi_{S}\right) .
\end{aligned}
$$

$\left|S_{\perp}\right|$ is the average target polarization and $N^{\uparrow(\downarrow)}$ represent the luminosity normalized yields for the two polarized target helicity states. $\phi$ and $\phi_{S}$ are the azimuthal angles of the $\rho^{0}$ production plane and the transverse component of the target spin with respect to the lepton scattering plane, respectively.

Figure 2 displays the preliminary HERMES results for the $\sin \left(\phi-\phi_{S}\right)$ moment, $A_{U T}^{\sin \left(\phi-\phi_{S}\right)}$, of the asymmetry as a function of $x_{B}$. The data were taken during 2002-2004 on a transversely polarized hydrogen target with $\left|S_{\perp}\right|=0.76 \pm 0.05$. The moment is corrected for non-exclusive and non-resonant background contributions, however no longitudinal/transverse separation has

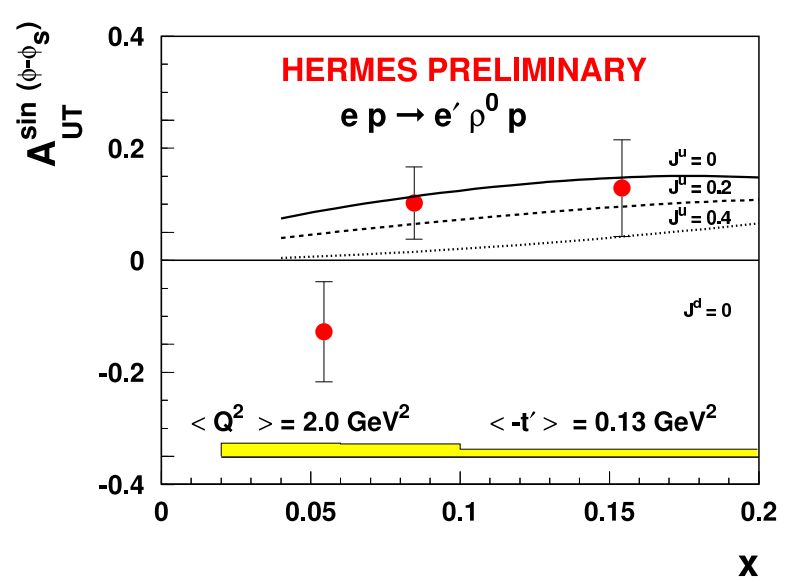

Fig. 2. The $\sin \left(\phi-\phi_{S}\right)$ moment of the exclusive $\rho^{0}$ TTSSA as a function of $x_{B}$, compared to GPD-based calculations [8].
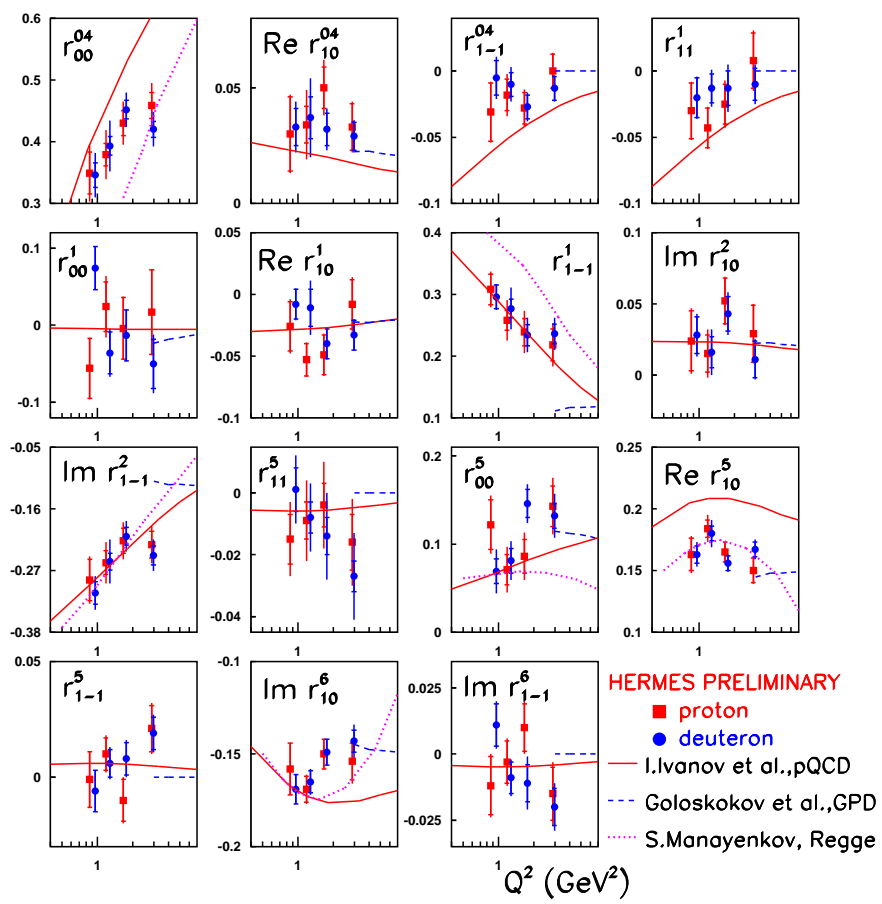

Fig. 3. The $Q^{2}$-dependence of the 15 unpolarized SDMEs for the proton and deuteron compared to theoretical calculations $[10-12]$.

been performed yet. The observed $x_{B}$-dependence agrees with the behavior predicted by GPD models [7]. In the figure the data are compared to GPD-based calculations for different values of the quark total angular-momentum contribution [8].

Another set of observables in exclusive $\rho^{0}$ production are the Spin Density Matrix Elements (SDMEs), which describe the helicity transfer from the virtual photon to the vector meson and can be accessed through measurements of angular distributions of the meson and its decay pions [9]. With the polarized HERA lepton beam, and for both unpolarized hydrogen and deuterium targets, the full set of 15 unpolarized plus 8 beam polarization-dependent 
SDME was determined via a maximum likelihood fit of Monte Carlo events to the measured data.

Figure 3 displays the unpolarized SDMEs for the proton and deuteron as a function of $Q^{2}$. A clear violation of the $s$-channel helicity conservation hypothesis is observed through, e.g., $r_{00}^{5}$ and Re $r_{10}^{04}$ being significantly non-zero. The data are compared to calculations based on pQCD $k_{T}$ factorization [10], Regge phenomenology [11] and on a GPD-based model [12]. Although a reasonable description of the data is obtained, these models are primarily based on Pomeron exchange at high energies and do not include quark exchange, which at HERMES kinematics is believed to be the dominant production mechanism [13].

\section{Hard exclusive pion pair production}

Hard exclusive electroproduction of $\pi^{+} \pi^{-}$pairs off nucleons represents another probe for the GPD $H$ and $E$. The process can proceed via $q \bar{q}$ or two-gluon exchange. In the former case the pair is produced with either $\rho$ meson (strong isospin $I=1$, total angular momentum $J=1,3 \ldots$ and $C$-parity $C=-1)$ or $f$-meson $(I=0$, $J=0,2 \ldots, C=+1)$ quantum numbers. The $C=+1$ $(C=-1) q \bar{q}$ exchange is described by flavour singlet (nonsinglet) parton combinations and due to $C$-parity conservation the pion pairs have $C=-1(C=+1)$ [14]. In the two-gluon exchange channel only $\rho$-meson-like pion pairs are created. Since this process allows one to distinguish two-gluon and $q \bar{q}$ exchange, new information can be gained on both the nucleon quark and gluon GPDs [15]. Furthermore, the interference between the two isospin channels may reveal information on the amplitude level of the weak isoscalar channel. To study the interference between the $P$-wave $(I=1)$ and $S, D$-wave $(I=0)$ production, the Legendre moments $\left\langle P_{1}(\cos \theta)\right\rangle$ and $\left\langle P_{3}(\cos \theta)\right\rangle$ were investigated [16]. These are defined as

$$
\left\langle P_{n}(\cos \theta)\right\rangle^{\pi^{+} \pi^{-}}=\frac{\int_{-1}^{1} \mathrm{~d} \cos \theta P_{n}(\cos \theta) \frac{\mathrm{d} \sigma^{\pi^{+} \pi^{-}}}{\mathrm{d} \cos \theta}}{\int_{-1}^{1} \mathrm{~d} \cos \theta \frac{\mathrm{d} \sigma^{\pi^{+} \pi^{-}}}{\mathrm{d} \cos \theta}}
$$

where $\theta$ is the polar angle of the $\pi^{+}$with respect to the direction of the $\pi^{+} \pi^{-}$pair in the center-of-momentum frame of the virtual photon and target nucleon. The production cross-section $\mathrm{d} \sigma^{\pi^{+} \pi^{-}} / \mathrm{d} \cos \theta$ can be expressed in terms of the spin density matrix $\rho_{\lambda \lambda^{\prime}}^{J J^{\prime}}$ of the pion pair, where the diagonal elements $\rho_{\lambda \lambda}^{J J}$ give the probability to produce the pion pair with angular momentum $J$ and projection $\lambda$ and the off-diagonal terms describe the interference terms. Contributions with $J>2$ are expected to be negligible in the range of the pion pair invariant mass $M_{\pi \pi}$ covered by HERMES. The two Legendre moments then become $\left\langle P_{1}\right\rangle=\frac{1}{\sqrt{15}}\left[4 \sqrt{3} \rho_{11}^{21}+4 \rho_{00}^{21}+2 \sqrt{5} \rho_{00}^{10}\right]$ and $\left\langle P_{3}\right\rangle=\frac{1}{7 \sqrt{5}}\left[-12 \rho_{11}^{21}+6 \sqrt{3} \rho_{00}^{21}\right]$, i.e. $\left\langle P_{1}\right\rangle$ is sensitive to the $P$-wave interference with $S$ - and $D$-waves, whereas $\left\langle P_{3}\right\rangle$ is sensitive to the $P$-wave interference with a $D$-wave only.

Figure 4 shows the measured $\left\langle P_{1}\right\rangle$ moment for a hydrogen target. At $M_{\pi \pi}$ values around the $\rho^{0}$ mass, the

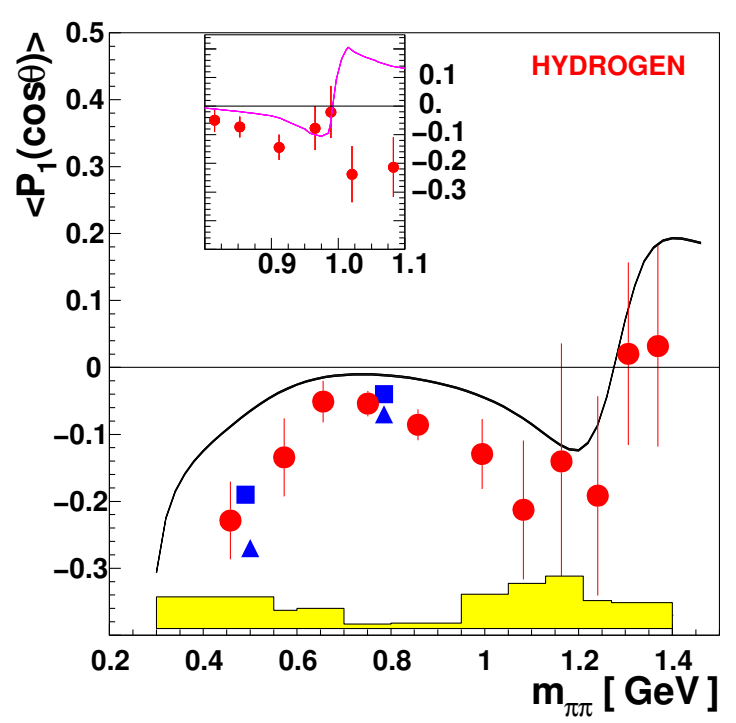

Fig. 4. The $M_{\pi \pi}$-dependence of the $\left\langle P_{1}\right\rangle$ moment for hydrogen data compared to leading-twist predictions including gluon exchange (solid curve) and without gluon exchange (squares at $x_{B}=0.1$ and triangles at $x_{B}=0.2$ ) [15]. The insert displays the $f_{0}(980)$ region with finer bins together with calculations including $f_{0}$ contributions [17].

absolute value of the moment shows a minimum, indicating the dominant role of $\rho^{0}$ production in the denominator of the moment. At higher and lower masses one can observe the interference of the $\rho^{0}$ tails with the non-resonant $S$-wave amplitude. At $M_{\pi \pi} \approx 1 \mathrm{GeV}$, one sees a hint of the interference of the $\rho^{0}$ tail and the $S$-wave $\pi^{+} \pi^{-}$production from the narrow $f_{0}(980)$-resonance. In the $f_{2}(1270)$ meson region a sign change is suggested due to the interference between the $\rho^{0}$ upper tail and the $f_{2} D$-wave. A fair agreement is found between the data and the GPDbased model predictions [15].

\section{References}

1. D. Müller et al., Fortschr. Phys. 42, 101 (1994); A.V. Radyushkin, Phys. Lett. B 380, 417 (1996); X. Ji, Phys. Rev. Lett. 78, 610 (1997).

2. J. Collins et al., Phys. Rev. D 56, 2982 (1997).

3. K. Ackerstaff et al., Nucl. Instrum. Methods A 417, 230 (1998).

4. M. Vanderhaeghen et al., Phys. Rev. D 60, 094017 (1999).

5. L. Mankiewicz et al., Eur. Phys. J. C 10, 307 (1999).

6. J.M. Laget, Phys. Rev. D 70, 054023 (2004).

7. K. Goecke et al., Prog. Part. Nucl. Phys. 47, 401 (2001).

8. F. Ellinghaus et al., hep-ph/0506264.

9. K. Schilling, G. Wolf, Nucl. Phys. B 61, 381 (1973).

10. I.P. Ivanov et al., hep-ph/0501034, DESY-04-243.

11. S.I. Manayenkov, Eur. Phys. J. C 33, 397 (2004).

12. S.V. Goloskokov, P. Kroll, Eur. Phys. J. C 42, 281 (2005).

13. M. Diehl, A.V. Vinnikov, Phys. Lett. B 609, 286 (2005).

14. M. Diehl, Phys. Rep. 388, 41 (2003).

15. B. Lehmann-Dronke et al., Phys. Rev. D 63, 114001 (2001); Phys. Lett. B 475, 147 (2000).

16. A. Airapetian et al., Phys. Lett. B 599, 212 (2004).

17. Ph. Hägler et al., Eur. Phys. J. A 18, 389 (2003). 